\title{
The Design of Vehicle Antenna Servo Control System Based on Ziegler Nichols PID Control
}

\author{
Yueming Dai ${ }^{1, a}$, Rui Zhang ${ }^{2, b}$ \\ ${ }^{1}$ Department of Information and Electronic Engineering,Xuzhou Institute of Technology, Xuzhou , \\ 221111 China \\ ${ }^{2}$ School of Science, QingdaoTechnological University, Qingdao 266033 , China \\ aemail: daiym63@163.com, bemail: daiyu2@sina.com
}

Keywords: Adaptive PID control; Ziegler and Nichols parameter tuning; Vehicle servo system; Critical stable

\begin{abstract}
In this paper, a method based on Ziegler Nichols - frequency response of the vehicle servo system adaptive PID controller is proposed. The control circuit online identify important critical frequency response characteristics of process object through input and output data in the normal process operation, and then the system online update PID controller parameters based on Ziegler Nichols setting rules or by the improved method. The adaptive process of PID controller can online real-time correct parameters and ensure the control loop always work in the best condition even under the operating conditions change.The results show that the method has stronger robustness and adaptive ability.
\end{abstract}

\section{Introduction}

The traditional PID control principle is simple for use and strong adaptability, so it widely used in all kinds of industrial process control field. But PID controller parameters adjustment needs certain process and the optimal parameters selection is more troublesome, the process of PID control can't effectively to online control system for some system parameters change, so it can't satisfy the PID parameter changes request with the corresponding system parameters change, that it seriously affected the control effect. Ziegler - Nichols parameters tuming method is proposed in this paper: when the process variable and the speed change, we establish a set of empirical formula that set these test results into the right performance parameters. Ziegler - Nichols frequency response method proposed in this paper online optimize the feedback rotor speed and magnetic pole position of vehicle antenna servo system, improve the control precision of the system,and can guarantee the system always run in the best state. Through the establishment of motor rotating speed model, we constructed vehicle servo control system simulation diagram based on PID tuning Ziegler - Nichols method.The simulation results show that the Ziegler Nichols parameter tuning method PID controller has good dynamic performance on tracking the set point response, less overshoot and good steady state performance. It has a strong ability to adapt to the object parameters change.

\section{The mathematical model of vehicle antenna servo system}

Vehicle antenna system consists of two parts: indoor equipment and outdoor equipment. Outdoor equipment is mainly antenna servo tracking system (including the platform, platform servo tracking system, inertial sensors, GPS, satellite antenna, etc.); Indoor equipment is mainly the controller (including the sensor interface, data acquisition, controllers, satellite receiver, etc.) and the host computer.Two parts use cable connection. The system has the two big functions of a stable track and recept satellite singnals. This system uses single-chip microcomputer control and takes only less hardware system, then motor control system can be realized. This system adopts the incremental light code disc feedback the rotor speed and magnetic pole position and the initial position. Vehicle antenna servo system control diagram is shown in figure1. 


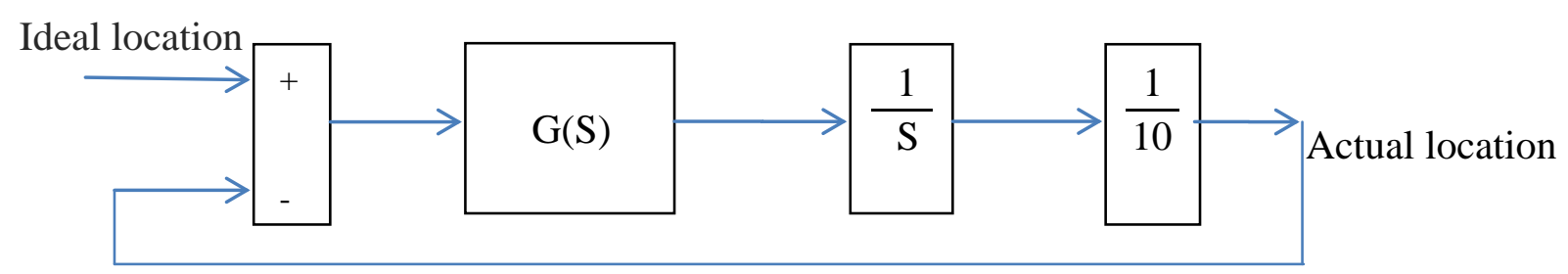

Fig.1 DC servo motor rotation system structure diagram(1/10 for worm and gear reduction ratio)

If the motor load is constant and only the output of motor rotation angular velocity, then get the transfer function of the dc servo motor type as follows[1]:

$$
G(S)=\frac{\omega \mathrm{a}(\mathrm{S})}{V a(S)}=\frac{\mathrm{Kt} / \mathrm{LaJ}}{\left.S^{2}+[(\mathrm{RaJ}+\mathrm{LaB}) / \mathrm{La}] \mathrm{S}+[(\mathrm{RaB}+\mathrm{Kt} \mathrm{Kv})] / \mathrm{La}\right]}
$$

Among formula (1), wa is rotor rotating angular speed, Ra. La are magnetic flux density and the rotor winding number of permanent magnet ; Kv; Kt are the rate constant and torque constant determined by the physical properties of iron core, $\mathrm{J}$ is the rotational inertia of the rotor and the motor load. B is the damping constant of the mechanical rotating system. By the motor features and system features, we get motor parameters Ra; La; Kv; Kt. J value. The parameter values replaced in formula (1) and we get the transfer function of the motor as shown in the following formula:

326.5

$$
G(S)=\overline{S^{2}+618.4 S+61.28}
$$

\section{Ziegler Nichols method to realize the PID parameter tuning}

$$
\begin{aligned}
\Delta u(k) & =u(k)-u(k-1) \\
& =K_{P}[e(k)-e(k-1)]+K_{I} e(k)+K_{D}[e(k)-2 e(k-1)+e(k-2)] \\
u(k) & =K_{P}\left\{e(k)+\frac{T}{T_{I}} \sum_{j=0}^{k} e(j)+\frac{T_{D}}{T}[e(k)-e(k-1)]\right\}
\end{aligned}
$$

Formula (3) is called incremental PID control formula; Formula (4) is called position type PID control equation. Kp is proportional 'coefficient, $K_{I}=K_{P} \frac{T}{T_{I}}$ is integral coefficient and $K_{D}=K_{P} \frac{T_{D}}{T}$

is differential coefficient. Because position type PID controller adopts total output, it's output is the actual position of the actuator and each output is associated with the past state and calculation of deviation accumulation. So large amount of calculation work must do, once the data processing computing chips appear problem, the system will make the output volatility, and result in high volatility in the actuator, that causes big accidents.

In incremental algorithm, the proportional and integral item symbols have the following relations: if the controlled variable to continue deviates from the given value, the two symbols are the same; and when the controlled variable to the given value direction changes, the two symbols instead. This algorithm can make the dynamic process of control system accelerate. The incremental PID algorithm is better.

Ziegler Nichols method may determine very precise parameters for controller, it also can fine-tune after this.(1) Build a closed loop control circuit, determine the stability limit;(2)According to the formula to calculate the controller parameters[2].The stability limit is determined by the $\mathrm{P}$ component. When there is a steady oscillation to reach this limit, the critical coefficient Kpcrit and critical oscillation cycle Tcrit are generated. After determining the critical coefficient Kpcrit and critical oscillation period Tcrit, we calculate other parameters according to a formula in the table1 below . 
Table1 PID parameters calculating formula

\begin{tabular}{|c|c|c|c|c|c|}
\hline \multirow{2}{*}{$\begin{array}{c}\text { The controller } \\
\text { type }\end{array}$} & \multicolumn{5}{|c|}{ characteristic data } \\
\cline { 2 - 6 } & $K_{P}$ & $T_{I}$ & $T_{D}$ & $K_{i}$ & $K_{D}$ \\
\hline PID & $0.6 K_{\text {Pcrit }}$ & $0.5 T_{c r i t}$ & $0.12 T_{c r i t}$ & $\frac{K_{P}}{T_{I}}$ & $K_{P} T_{D}$ \\
\hline
\end{tabular}

For the transfer function of a given controlled object,we select the intersection point between root locus diagram of discrete system and z plane unit circle on the root locus diagram,then we gain gain $\mathrm{Km}$ and this point $\omega$ value namely $\omega_{m}$. Tuning formula is as follows:

$$
K_{p}=0.6 K_{m}, K_{d}=\frac{K_{P} \pi}{4 \omega_{m}}, K_{i}=\frac{K_{p} \omega_{m}}{\pi}
$$

In formula(5), $\mathrm{Km}$ is the $\mathrm{Kp}$ values of the system initiate oscillation, $\omega_{m}$ for the oscillation frequency. Oscillation frequency $\omega_{m}$ can be determined by the pole Angle $\theta$ located on the unit circle, $\omega_{m}=\theta / \mathrm{T}$ ( $\mathrm{T}$ for the sampling period) [3].

Take sampling period: $\mathrm{T}=0.25 \mathrm{~s}$

The amplifier amplification coefficient: $\mathrm{Kp}=100$

By the given dynamic structure diagram, the open-loop transfer function is formula(6):

$G(S)==\frac{326.5}{S^{2}+618.4 S+61.28} * \frac{1}{S} * \frac{1}{10}$

The Ziegler Nichols PID parameter tuning operation result is shown in figure 2 .

We select red point in figure 2 and click the left mouse button, then the computing results are obtained.

selected_point pole $=-2.0447+1.7221 \mathrm{i} ;-2.0447-1.7221 \mathrm{i} ;-0.0001$

$\mathrm{kp}=2.2523 \mathrm{e}+003 ; \mathrm{kd}=181.1246 ; \mathrm{ki}=7.0019 \mathrm{e}+003$

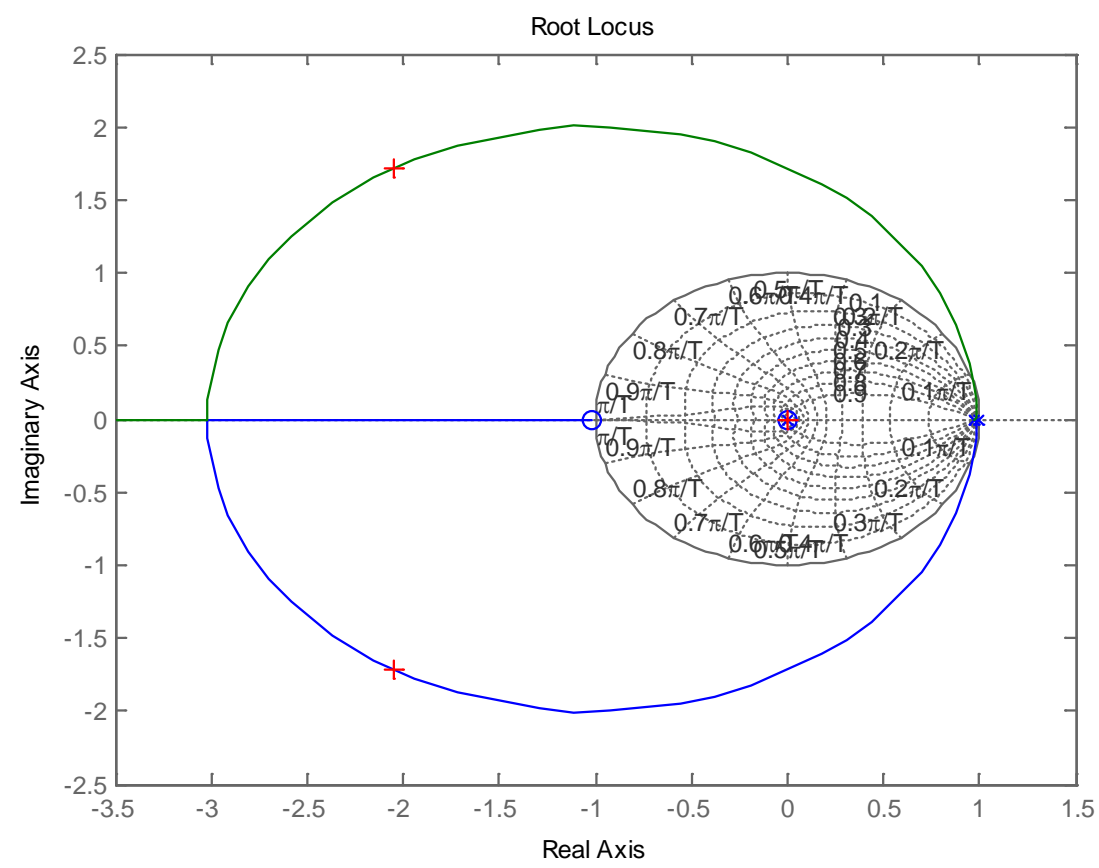

Fig. 2 Root locus diagram of program 


\section{The Simulink simulation}

(1) To construct system of Simulink model[4][5]:

The simulink model of vehicle servo system with adaptive PID controller is shown in figure 3.

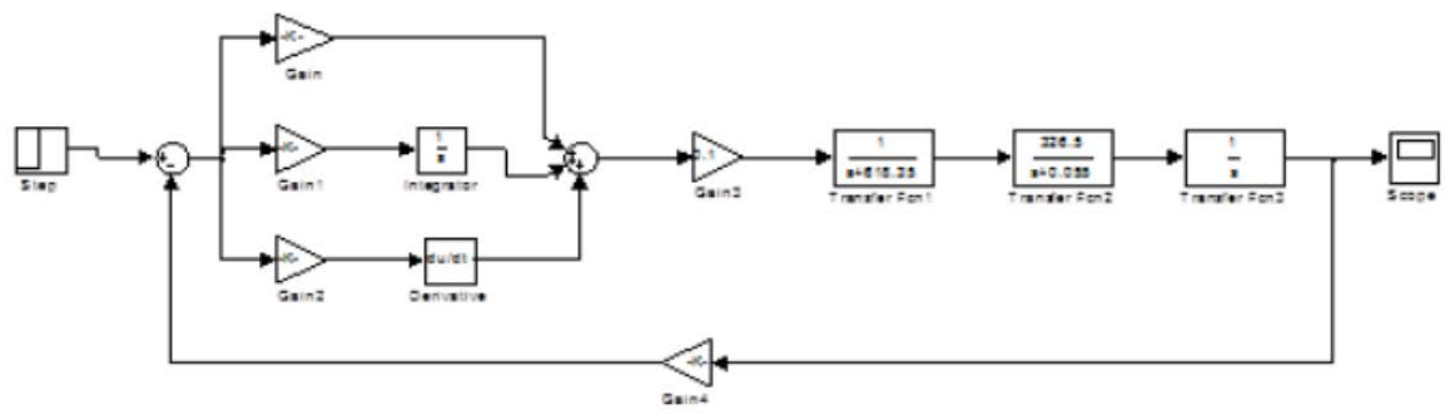

Fig.3 System simulation model

(2) The results of simulation:

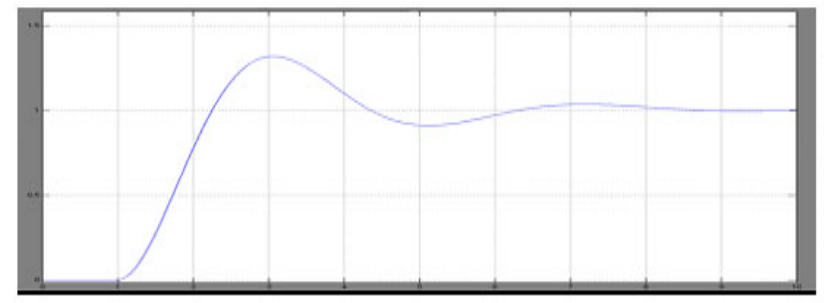

Fig. 4 Traditional PID dynamic response

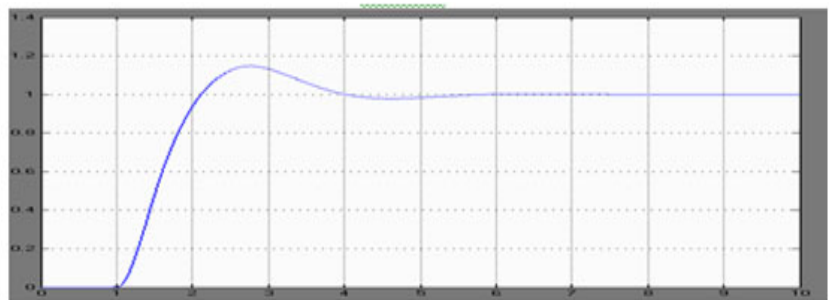

Fig.5 Adaptive PID dynamic response based on Ziegler Nichols - frequency method

The simulation results are shown in figure 5. Compared with the traditional PID simulation figure 4, we know that the rise time and adjust the time of the system greatly lessen, overshoot significantly reduce, greatly improving the dynamic performance of the system under the step response.

\section{Conclusion}

This design designed a reasonable Ziegler - Nichols PID adaptive controller and MATLAB simulation for a vehicle servo system. Because the vehicle antenna is in a real-time change of environment, it may lead to system parameters change according to the environmental changes. The traditional fixed parameters control strategy is no way to meet this demand, and Ziegler - Nichols adaptive control may compensate this faultiness. Ziegler - Nichols adaptive control also is very good solution to the servo system itself own error caused by inertia. Ziegler - Nichols methods are method of PID tuning based on system stability analysis. Ziegler - Nichols method can quickly and accurately calculate the corresponding parameter numerical, then only need to fine-tune the ideal control effect can be got.Software and hardware provide reference for military radar antenna design combined with satisfy the system real fast, accurate, stable.

\section{Reference}

[1] Tang Jie, motor and drag (second edition), higher education press, 2007.

[2] li-li hu; Ya-gang wang;Hu li; The PID self-tuning algorithm based on data-driven study [J]; Electronic technology; 01, 2013.

[3] Rutao pan; and the PID controller parameter setting method [J]; Science and technology information; 07, 2010.

[4] Zhao Jingbo, MATLAB simulation and control system design, mechanical industry publishing house, 2010.

[5]Liu Jinkun, advanced PID control MATLAB simulation, the electronic industry press,2011. 